\title{
Estado actual de la utilización de la hemodiálisis domiciliaria: revisión integrativa
}

\author{
Ana Belén Romero Ruiz ${ }^{1}$, Elizabeth Serrano Madero' ${ }^{1}$, Rodolfo Crespo Montero ${ }^{1,2,3}$ \\ ${ }^{1}$ Departamento de Enfermería. Facultad de Medicina y Enfermería. Universidad de Córdoba. España \\ 2 Unidad de Gestión Clínica de Nefrología. Hospital Universitario Reina Sofía de Córdoba. España \\ ${ }^{3}$ Instituto Maimónides de Investigación Biomédica de Córdoba. España
}

\section{Resumen}

Objetivo: Conocer la producción científica existente sobre el estado actual de la hemodiálisis domiciliaria.

Material y Método: Se ha realizado una revisión integrativa mediante una búsqueda en las bases de datos Pubmed, Scopus y Google Académico. Se incluyeron artículos científicos originales y de revisión, en inglés y español, excluyendo aquellos artículos que no presentaban resultados o que no estuviesen en texto completo. Resultados: Se han incluido 23 artículos publicados entre el año 2012 y 2017. La hemodiálisis domiciliaria es poco utilizada en el mundo, a excepción de Australia y Nueva Zelanda. Las series publicadas encuentran mejor supervivencia en los pacientes en hemodiálisis domiciliaria diaria o intensiva, respecto a otras modalidades de terapia sustitutiva renal, así como mejores resultados clínicos. Como principales ventajas de la técnica, se destaca la flexibilidad en la vida diaria y la independencia del centro sanitario. Como principal inconveniente se ha encontrado un aumento de las infecciones relacionadas con el uso de catéteres venosos centrales o la técnica de punción en "ojal".

Conclusiones: La hemodiálisis domiciliaria presenta como principal ventaja la mayor libertad y flexibilidad para que el paciente dirija su propio tratamiento, siendo el principal inconveniente, una mayor probabilidad de padecer infecciones, relacionadas con el acceso vascular. Cuando se realiza hemodiálisis domiciliaria

\section{Correspondencia:}

Ana Belén Romero Ruiz

C/ General Lázaro Cárdenas Mz 5, 3, 2 3. 14013 Córdoba E mail:n32rorua@uco.es diaria o intensiva los resultados clínicos se muestran superiores a los otros procedimientos de diálisis, siendo comparables a los del trasplante renal. El papel de enfermería es fundamental en el desarrollo de esta técni$\mathrm{ca}$, especialmente en el proceso clave de la enseñanza al paciente y familiar.

PALABRAS CLAVE: hemodiálisis domiciliaria; supervivencia de hemodiálisis en casa; formación de enfermería.

\section{Current state of use of domiciliary hemodialysis: An integrative review}

\section{Abstract}

Objective: To know the existing scientific literature on the current state of home hemodialysis.

Material and Method: An integrative review has been carried out through a search of Pubmed, Scopus and Google Academic databases. Original scientific and review articles were included, in English and Spanish, excluding those articles that did not present results or that were not in full text.

Results: Twenty-three articles published between 2012 and 2017 have been included. Home hemodialysis is rarely used in the world, apart from Australia and New Zealand. The published series reported better survival in patients on daily or intensive home hemodialysis, compared to other types of renal replacement therapy, as well as better clinical results. The main advantages 
of the technique are the flexibility in daily life and the independence of the health center. As main drawback, an increase in infections associated with the use of central venous catheters or buttonhole puncture technique was shown.

Conclusions: Home hemodialysis has the main advantage of greater freedom and flexibility for the patient, for the management of their own treatment. The main drawback is a higher probability of suffering infections, related to vascular access. When daily or intensive home hemodialysis is performed, the clinical results are better than the other dialysis procedures, being comparable to those of kidney transplantation. The role of nursing is fundamental in the development of this technique, especially in the key process of patient and family education.

KEYWORDS: home hemodialysis; survival of home hemodialysis; nursing training.

\section{Introducción}

La enfermedad renal crónica constituye hoy día, un importante problema de salud pública. La manifestación más avanzada, la enfermedad renal crónica avanzada (ERCA) en estadio 5 y la consiguiente necesidad de tratamiento renal sustitutivo (TRS) mediante diálisis peritoneal (DP), hemodiálisis (HD) o trasplante renal (TR), presenta una incidencia y una prevalencia crecientes en las últimas décadas ${ }^{1-5}$.

Según el Registro de 2015 de Diálisis y Trasplantes, de la Sociedad Española de Nefrología, la prevalencia de pacientes en TR es del 52,5\%, en HD es del $41,9 \%$ y en DP $5,5 \%$, en nuestro país. La mayoría de los pacientes que presentan esta enfermedad tienen una edad comprendida entre los 65 hasta los 74 años, siendo la técnica de diálisis más prevalente, la $\mathrm{HD}^{6}$.

Este aumento progresivo de la incidencia y prevalencia de la ERCA y la necesidad de TRS no es exclusivo de España. En efecto, la prevalencia de la ERCA ha aumentado progresivamente en todo el mundo y los registros de todos los países que poseen datos, muestran un continuo crecimiento de pacientes que necesitan TRS ${ }^{7}$, tal como muestra el registro de la European Dialysis and Transplant Association ${ }^{6,8}$.
Dentro de la técnica de HD, una opción terapéutica poco utilizada es la HD domiciliaria (HDD). La HDD es una alternativa de tratamiento, que permite flexibilizar los esquemas dialíticos convencionales de 3 veces a la semana, haciéndola más frecuente y/o más prolongada, ya que se realiza en casa del paciente. De esta manera se consigue optimizar resultados clínicos de una forma económicamente sostenible y mejorando la calidad de vida del paciente, al permitir una mayor flexibilidad en la terapia y mayor disponibilidad de tiempo libre?

Además, la HDD se asocia con otros efectos beneficiosos, permitiendo su uso diario con reducción de costos. Sin embargo, presenta una serie de contraindicaciones que se deben tener en cuenta a la hora de considerar a un paciente candidato ${ }^{10}$.

En España, la HDD tiene un escaso desarrollo, alcanzando tan solo el $0,3 \%$ del total de los pacientes en diálisis ${ }^{11}$. Por el contrario, la prevalencia de la técnica ha sido siempre elevada en Australia y Nueva Zelanda $(9,1 \%$ y $19 \%$, respectivamente), existiendo un aumento progresivo en países del norte de Europa como Dinamarca $(5,7 \%)$, Finlandia $(5,3 \%)$ o Reino Unido (4\%), donde los esfuerzos por su desarrollo están siendo muy importantes. Hay que destacar también Canadá, con una prevalencia del 3,9\%, desde donde surgen variadas publicaciones cientí-ficas, y Estados Unidos $(1,3 \%)$, con gran crecimiento desde el año $2002^{12}$. Aunque las cifras globales siguen siendo todavía muy bajas, el crecimiento en los últimos tiempos ha sido muy importante y se espera un mayor desarrollo de la técnica en los próximos años, con crecimiento progresivo en incidencia y prevalencia de pacientes.

Según diversos autores, una forma de potenciar esta técnica es estableciendo estrategias basadas en la promoción de la HDD en nuestro medio y abordar de forma realista y a un coste asumible los retos que plantean las distintas opciones de diálisis; así como proporcionando tranquilidad al paciente a la hora de abordar los posibles problemas que se le presenten en casa y la implicación de las instituciones, ya que en la actualidad es el único tipo de HD que permitiría reducir el intervalo dialítico en muchos pacientes ${ }^{11-13}$.

Sin embargo, dada la escasa población que recibe HDD, parece interesante revisar la bibliografía actual acerca de esta técnica, analizando las indudables ventajas que ofrece, las dificultades para su implementación, así como el rol de enfermería en el desarrollo de la misma. El papel de enfermería es fundamental en el 
desarrollo de esta modalidad de HD, pues la formación $y$ el entrenamiento de pacientes y familiares para llevar a cabo la HDD, es una competencia propia de enfermería, así como proporcionar apoyo tanto al inicio de la técnica como en el seguimiento de la misma ${ }^{14}$.

Con el objetivo general de conocer la producción científica existente sobre el estado actual de la HDD, nos planteamos los siguientes objetivos específicos:

- Describir las ventajas y desventajas del uso de esta técnica.

- Analizar los resultados clínicos de la técnica.

- Valorar el papel del personal de enfermería en la implantación y desarrollo de la HDD.

\section{Material y Método}

El diseño empleado fue el de un estudio de revisión integrativa, método que permite conocer el estado actual de un fenómeno de estudio, identificar la necesidad de futuras investigaciones y detectar los puntos importantes del estudio. En este tipo de revisiones, los resultados se obtienen mediante la integración de conceptos que son extraídos a partir del uso de distintos enfoques metodológicos, incluyendo experimentales y no experimentales, así como experiencias prácticas y análisis de política $^{15,16}$, de acuerdo a las siguientes fases ${ }^{16}$ :

1. Identificación del objetivo de la revisión: ¿Cuál es el estado actual de la utilización de la HDD?

2. Determinación de los criterios de búsqueda: la búsqueda se realizó en diversas bases de datos con el objetivo de recopilar la mayor producción posible disponible en relación con el tema a tratar, en los últimos 5 años (entre 2012 y 2017). Las bases de datos empleadas fueron: Pubmed, Scopus y Google Académico. El periodo de consulta tuvo lugar desde Enero hasta Abril de 2017.

En la Tabla 1, se representan los artículos encontrados en la primera búsqueda y los seleccionados.

Tabla 1. Tabla de resultados de la búsqueda.

\begin{tabular}{lccc}
\hline Bases de datos & Pubmed & Scopus & Google académico \\
\hline $\begin{array}{l}\text { Total de publicaciones } \\
\text { localizadas }\end{array}$ & 116 & 35 & 142 \\
\hline $\begin{array}{l}\text { Total de publicaciones } \\
\text { seleccionadas }\end{array}$ & 9 & 1 & 13 \\
\hline
\end{tabular}

El método de búsqueda consistió en utilizar como palabras clave: hemodiálisis domiciliaria (Home hemodialysis), supervivencia en hemodiálisis domiciliaria (Home hemodialysis survival), formación enfermera (Nursing training). En cada base de datos se llevó a cabo estrategias de búsqueda diferentes, realizando distintas pruebas de asociación entre los descriptores anteriormente citados, teniendo en cuenta que cada uno de los descriptores empleados estuvieran presentes en el resumen, título del artículo y, si la base de datos lo presenta, en palabras clave, y de acuerdo a los siguientes criterios de inclusión: artículos originales, artículos de revisión, artículos en inglés y en español y estudio en humanos. Los criterios de exclusión fueron: artículos sin texto completo disponible o que no presentaban resultados.

3. Evaluación de los documentos incluidos en el estudio: al inicio de la búsqueda no se consideró ningún criterio de inclusión/exclusión en las diversas bases de datos, para así conocer la cantidad de producción científica existente en el tema y obtener mayor conocimiento del mismo, de manera que se aceptaron todos los trabajos que tratasen sobre el tema en cuestión. Tras la lectura de los títulos y resúmenes de todos los artículos, se fueron descartando aquellos que no cumplían con los criterios de inclusión y exclusión. Posteriormente se realizó la lectura completa de los trabajos seleccionados y se realizó un nuevo descarte de aquellos que no versaban sobre el tema a estudiar. Tras este proceso de cribado, el número de artículos total que cumplían con los criterios de inclusión marcados y que se han utilizado para el estudio han sido de 23. En ese momento se comenzó el análisis de los artículos recopilados.

4. Determinación de la información a obtener de los artículos: definición de HDD, el estado actual de la misma, las indicaciones para conocer los pacientes aptos para el uso de esta técnica, las ventajas e inconvenientes que presenta, el papel que tiene la enfermería en su desarrollo, la carga que supone para los cuidadores de los pacientes de HDD, los resultados clínicos que proporciona al paciente, la técnica de canulación y la supervivencia de HDD frente a otras modalidad de tratamiento.

5. Análisis del conocimiento: en esta fase se procedió a la interpretación de la información recopilada.

6. Síntesis de resultados: se llevó a cabo la integración de las conclusiones obtenidas. 


\section{Resultados}

\section{Selección de estudios preliminar}

De un total de 293 artículos preseleccionados, se excluyeron 195 durante la revisión de títulos y resúmenes. Otros 53 fueron eliminados tras la revisión del texto completo. Finalmente, se seleccionaron 23 artículos que cumplían con los criterios de inclusión/exclusión previamente establecidos, tal como se refleja en la Figura 1.

\section{Descripción de los resultados en función de las variables}

\section{Indicaciones}

No todos los pacientes que precisan TRS con HD son aptos para la HDD, debido a que no reúnen los requisitos necesarios (pacientes dependientes, pacientes con falta de acceso vascular adecuado, pacientes con trastornos hemorrágicos, pacientes con enfermedades mentales graves (psicosis), pacientes con condiciones higiénicas inadecuadas, déficit sensorial... $)^{10}$. Algunos autores, consideran que algunos de estos pacientes podrían llevar a cabo la HD en su domicilio, pero recibiendo el apoyo y la ayuda necesaria ${ }^{17}$.

\section{Ventajas e inconvenientes}

Según diferentes autores, las principales ventajas que se asocian al uso de la HDD son la mayor libertad y flexibilidad para que el paciente dirija su propio tratamiento. Algunos autores también manifiestan que esta opción terapéutica mejora las oportunidades de empleo y conlleva beneficios psicológicos y que algunos pacientes han mejorado las relaciones con la familia, amigos y cuidadores, ya que pueden participar más en las actividades cotidianas ${ }^{10,18}$. También se ha asociado esta técnica a una mejora de los resultados clínicos del paciente, que serán abordados en otra variable $e^{19,20,21}$.

Respecto a los inconvenientes cabe destacar, la falta de autoconfianza y/o autoeficacia para manejar la propia terapia tanto en el paciente como en los proveedores de atención renal, falta de apoyo familiar y social, temor a la punción, falta o insuficiente orientación y compromiso por parte de la administración sanitaria ${ }^{10,19,20,22}$. Sin embargo, un artículo ${ }^{19}$, destaca que estas limitaciones no deben ser percibidas como barreras, sino como posibles áreas de mejora, lo que en última instancia puede conducir a mayores oportunidades para que los pacientes puedan beneficiarse de las ventajas que la HDD ofrece.

Otro inconveniente, es sin duda, el riesgo de infección, puesto que sigue siendo una importante barrera para la implementación de HDD. En algunos estudios, la in-
Los datos más relevantes de cada estudio se muestran en la Tabla 2. 
Tabla 2. Artículos de estudio.

\begin{tabular}{|c|c|c|c|}
\hline $\begin{array}{l}\text { Autor, Año, } \\
\text { País }\end{array}$ & $\begin{array}{l}\text { Diseño del } \\
\text { estudio }\end{array}$ & Muestra & Principales conclusiones \\
\hline $\begin{array}{l}\text { Karkar A, et al. } \\
\text { 2015. Kingdom } \\
\text { of Saudi Arabia } \\
\text { (Dammam) (10). }\end{array}$ & Revisión & $\begin{array}{l}\text { No se espe- } \\
\text { cifica }\end{array}$ & $\begin{array}{l}\text { HDD es una modalidad que se percibe como beneficiosa. Se puede realizar de } \\
\text { forma segura e independiente en el hogar con mejoras significativas en los re- } \\
\text { sultados de salud y la utilización de recursos. Un programa bien establecido y } \\
\text { adecuadamente presupuestado para pacientes elegibles y adecuados, puede des- } \\
\text { empeñar un papel importante en la implantación y la continuidad de la HDD. }\end{array}$ \\
\hline $\begin{array}{l}\text { Tennankore K, et } \\
\text { al. 2014. Canadá } \\
\text { (17). }\end{array}$ & Revisión & $\begin{array}{l}\text { No se } \\
\text { especifica }\end{array}$ & $\begin{array}{l}\text { HDD intensiva tiene ventajas clínicas importantes. Si bien hay riesgos, también } \\
\text { presentan una oportunidad para las iniciativas de mejora de la calidad para opti- } \\
\text { mizar aún más la prestación de esta técnica. }\end{array}$ \\
\hline $\begin{array}{l}\text { Walker RC, et } \\
\text { al. 2016. Nueva } \\
\text { Zelanda (18) }\end{array}$ & $\begin{array}{l}\text { Estudio } \\
\text { cualitativo }\end{array}$ & $\begin{array}{l}43 \text { pacientes } \\
\text { y } 9 \\
\text { cuidadores }\end{array}$ & $\begin{array}{l}\text { Se sugiere mayor énfasis en el desarrollo de programas y recursos educativos } \\
\text { enfocados en el paciente. Los programas de diálisis en el hogar pueden reducir } \\
\text { los temores y generar más confianza. Las cargas financieras pueden minimizarse } \\
\text { mediante la prestación de programas de reembolso, el apoyo al empleo y la asis- } \\
\text { tencia adicional para los pacientes. }\end{array}$ \\
\hline $\begin{array}{l}\text { Tennankore KK, et } \\
\text { al. 2012. Canadá } \\
\text { (19). }\end{array}$ & Revisión & $\begin{array}{l}\text { No se } \\
\text { especifica }\end{array}$ & $\begin{array}{l}\text { HDD diaria intensiva presenta múltiples beneficios, que han llevado a un mayor } \\
\text { interés internacional; y se han identificado barreras específicas. Sin embargo, } \\
\text { estas limitaciones no deben ser percibidas como fallas, sino como áreas para } \\
\text { enfocarse y mejorar, lo que conduce a mayores oportunidades para los pacientes. }\end{array}$ \\
\hline $\begin{array}{l}\text { Trinh E, et al. } \\
\text { 2017. Canadá } \\
\text { (20). }\end{array}$ & Revisión & $\begin{array}{l}\text { No se } \\
\text { especifica }\end{array}$ & $\begin{array}{l}\text { Destaca el resurgir de la HDD, aunque todavía existen barreras para aumentar } \\
\text { su uso. Mejorar la educación de los pacientes y proveedores de atención médica } \\
\text { es una prioridad importante, pero se necesitan más investigaciones sobre estra- } \\
\text { tegias para reducir el fracaso de la técnica y mantener a los pacientes en HDD. }\end{array}$ \\
\hline $\begin{array}{l}\text { Walker RC, et } \\
\text { al. 2014. Nueva } \\
\text { Zelanda (21). }\end{array}$ & $\begin{array}{l}\text { Revisión } \\
\text { sistemática }\end{array}$ & 24 estudios & $\begin{array}{l}\text { Los pacientes y cuidadores perciben que la HDD ofrece la oportunidad de prospe- } \\
\text { rar; Sin embargo, expresan ansiedad y temor acerca del inicio de HDD. Reconocer } \\
\text { y abordar estas aprehensiones permite la realización de programas de HDD que } \\
\text { respondan a las necesidades, preocupaciones y prioridades de los pacientes. }\end{array}$ \\
\hline $\begin{array}{l}\text { Schachter ME, et } \\
\text { al. 2012. Canadá } \\
\text { (22). }\end{array}$ & Revisión & $\begin{array}{l}\text { No se } \\
\text { especifica }\end{array}$ & $\begin{array}{l}\text { HD intensiva ofrece múltiples ventajas clínicas en comparación con la terapia de } \\
\text { reemplazo renal convencional, aunque la adopción de tales modalidades sigue } \\
\text { siendo relativamente baja. Es necesario el avance tecnológico para el desarrollo } \\
\text { de las técnicas de HDD. }\end{array}$ \\
\hline $\begin{array}{l}\text { Poon CK, et al. } \\
\text { 2016. Canadá } \\
\text { (23). }\end{array}$ & Revisión & $\begin{array}{l}\text { No se } \\
\text { especifica }\end{array}$ & $\begin{array}{l}\text { La infección es una complicación importante en los pacientes con HDD. Deben } \\
\text { hacerse esfuerzos para reducir su ocurrencia. La elección del acceso de diálisis } \\
\text { y la técnica de canulación debe ser individualizada. La revisión de la técnica y } \\
\text { las visitas domiciliarias desempeñan un papel importante para la prevención de } \\
\text { infecciones. }\end{array}$ \\
\hline $\begin{array}{l}\text { Tejada-Tayabas } \\
\text { LM, et al. } 2015 . \\
\text { Mexico (24). }\end{array}$ & $\begin{array}{l}\text { Estudio } \\
\text { cualitativo }\end{array}$ & $\begin{array}{l}9 \text { enferme- } \\
\text { ras }\end{array}$ & $\begin{array}{l}\text { Este estudio muestra la importancia y necesidad de establecer una estrategia } \\
\text { dirigida a los cuidadores, integrándolos en el grupo multidisciplinario de profe- } \\
\text { sionales de la salud que brindan atención a estos pacientes en el hospital para } \\
\text { establecer una atención coordinada hospital- adherencia terapéutica, eficacia de } \\
\text { sustitución de tratamiento y calidad de vida del paciente. }\end{array}$ \\
\hline $\begin{array}{l}\text { Tong A, et al. } \\
\text { 2013. Italia. } \\
\text { (25). }\end{array}$ & $\begin{array}{l}\text { Estudio } \\
\text { cualitativo }\end{array}$ & $\begin{array}{l}20 \\
\text { cuidadores } \\
22 \text { pacientes }\end{array}$ & $\begin{array}{l}\text { Los pacientes sin experiencia previa sobre la HDD y sus cuidadores reconocen } \\
\text { la autonomía de la HDD, pero están muy preocupados por la carga potencial y el } \\
\text { sacrificio personal que impone a los cuidadores y se sienten aprensivos en aceptar } \\
\text { las responsabilidades de la diálisis. Para promover la aceptación se necesitan } \\
\text { estrategias eficaces que proporcionen información y facilitar el apoyo de los pa- } \\
\text { cientes. }\end{array}$ \\
\hline $\begin{array}{l}\text { Hanson CS, et al. } \\
\text { 2016. Australia } \\
\text { (26). }\end{array}$ & $\begin{array}{l}\text { Estudio } \\
\text { cualitativo }\end{array}$ & 20 pacientes & $\begin{array}{l}\text { El entrenamiento en HDD fomenta la confianza en los pacientes; Sin embargo, } \\
\text { muchos experimentan estrés debido al aislamiento médico, responsabilidades de } \\
\text { tratamiento, imposiciones familiares y dificultades financieras. Abordar las preo- } \\
\text { cupaciones psicosociales del paciente puede aliviar las cargas en los pacientes y } \\
\text { familias durante la transición a la HDD. }\end{array}$ \\
\hline
\end{tabular}




\begin{tabular}{lll}
\multicolumn{1}{c}{$\begin{array}{c}\text { Autor, Año, } \\
\text { País }\end{array}$} & $\begin{array}{c}\text { Diseño del } \\
\text { estudio }\end{array}$ & \multicolumn{1}{c}{ Muestra }
\end{tabular}

Appleby S, et al.

(28).

piloto

pacientes
2013. Reino Unido Estudio 35

El proyecto de atención de 'cuidados compartidos', con la ayuda de la enfermera y la colaboración del paciente, mejoró los estándares clínicos y la elección del paciente con recursos mínimos. Esto ha resultado en 7 pacientes adicionales que realizan HDD y $17 \%$ de pacientes en el centro que ahora asumen la responsabilidad de su propia atención.

Este estudio describe las necesidades y preocupaciones de los cuidadores de la HDD. Los cuidadores tuvieron experiencias positivas y pudieron obtener ayuda cuando fue necesario en sus hogares. Los resultados indican, sin embargo, una necesidad de cuidado de relevo.

Las posibles mejoras en el diseño para mejorar la calidad y la seguridad de la experiencia del paciente incluyen características para ayudar a los pacientes a administrar sus servicios de diálisis para apoyar la comunicación entre las familias y los profesionales.

Estrategias adaptativas de afrontamiento se asocian con la mejora de la capacidad de sostener la terapia de HDD. Se necesita más investigación para determinar si la institución de estas intervenciones psicológicas ayudará a los pacientes a adoptar y mantener terapias de diálisis que requieren una mayor autogestión del paciente.

La canulación del ojal se asoció con mayor tasa de eventos infecciosos, aumento de los requisitos de apoyo del personal y ninguna reducción en las intervenciones quirúrgicas de la fístula arteriovenosa. Una revisión sistemática de la literatura publicada encontró que el ojal se asocia con mayor riesgo de infecciones arteriovenosas relacionadas con la fístula.

HDD intensiva es una modalidad óptima. Los profesionales de la salud pueden mejorar la atención, los resultados, creencias y competencias. Se necesitan cambios en las políticas y estructuras de financiación para desbloquear tanto el crecimiento de la HDD intensiva como la calidad de la atención y los resultados dentro del espacio clínico de HDD.

HDD: 1873

pacientes

al. 2012. EE.UU

(35).

Tennankore KK, et al. 2014. Canadá

(36).

Nesrallah GE, et al. 2016. Canadá (37).
HD en cen-

tro: 9365

pacientes

cohortes

Estudio de cohortes re- HDD: 173 trospectivo pacientes

Estudio de cohortes retrospectivo

3142

pacientes

Estudio de

4201 cohortes

pacientes

En relación con la hemodiálisis en el centro tres veces por semana, la hemodiálisis diaria en el hogar se asocia con modestas mejoras en la supervivencia.

El trasplante de riñón se asoció con un tratamiento superior y la supervivencia del paciente, pero hubo mayores tasas de hospitalización temprana, en comparación con HDD intensiva.

La hemodiálisis diaria en el hogar se asoció con menor riesgo de mortalidad que la diálisis peritoneal domiciliaria.

En relación con la DP, la HDD diaria se asocia con una disminución de la mortalidad, la hospitalización y el fracaso de la técnica. Sin embargo, los riesgos para la mortalidad y la hospitalización fueron similares con estas modalidades en los nuevos pacientes en diálisis. Se necesita más investigación.

Estudio de 11416 entre cohortes DP y HDD
HDD se asoció con una supervivencia superior del paciente en comparación con diálisis peritoneal.
AC, et al. 2015.

Australia y Nueva Zelanda (39). 
fección ocupa el primer lugar entre todas las causas de ingreso de los pacientes en HDD $(35,8 \%$ del total de ingresos) y una mayoría de estas infecciones están relacionadas con el acceso vascular ${ }^{23}$. En este artículo, además se encontró una alta tasa de bacteriemia relacionada con el catéter, en pacientes que iniciaban la HDD con un catéter venoso central (CVC), aunque sin diferencias significativas en comparación con los pacientes que se dializaban en centro. El uso de catéteres venosos centrales y la canulación con la técnica del "ojal" (Buttonhole) son factores de riesgo modificables importantes para la infección asociada a HDD, que se podrían aminorar con el desarrollo de una serie de medidas preventivas ${ }^{23}$.

\section{Papel de enfermería}

Según diversos artículos, enfermería desempeña un papel muy importante, ya que sobre ella recae la mayor responsabilidad en la formación del paciente, teniendo un contacto cercano y continuo con el mismo. De ahí, que la mayoría de los autores ponen el énfasis en la necesidad de una educación continua y orientación para el paciente ${ }^{18,24}$. De hecho, algunos autores destacan que la educación específica sobre la HDD puede aumentar la aceptación del paciente y captación en regiones que no disponen de ella ${ }^{25,26}$. En el proceso de formación, es fundamental la comprensión del manejo exitoso del acceso vascular por parte del paciente, puesto que supone el reto más importante de todo el procedimiento de diálisis, tanto en el hospital como en el hogar $20,27,28$.

\section{Carga del cuidador}

La carga del cuidador se propone como otro factor importante a tener en cuenta para el éxito de la HDD. En la mayoría de los casos, familiares no remunerados son los que asumen los cuidados del paciente (instalación, desmontaje y limpieza de la máquina). En algunos cuidadores, se detecta que en su mayoría viven situaciones de tensión, estrés, aparecen síntomas depresivos... descargar a los cuidadores, la disponibilidad de la línea de ayuda telefónica las 24 horas/día²9,30.

\section{Utilización de la técnica}

Dada la variabilidad del uso de la HDD, el porcentaje de utilización de esta técnica es muy dispar entre países. Según los datos existentes, esta técnica está implementada principalmente en Australia y Nueva Zelanda, donde la prevalencia de la HDD oscila entre el 9 y el 18 $\% 31$, sin que existan unas causas claras del porqué de su utilización en mayor o menor medida entre unos países y otros, al margen de las grandes distancias geográficas existentes en estos 2 países.

Recientemente ha aparecido en el mercado un monitor, el NxStage System One, que simplifica al máximo el tratamiento de HDD y por tanto, el manejo de la misma. En este no es necesario disponer de un tratamiento previo del agua y por tanto reduce mucho la necesidad de espacio físico32.

\section{Técnica de canulación}

La técnica de canulación mas utilizada en el paciente en HDD es la técnica del "ojal" 23,33. Esta técnica de punción se ha asociado a mayor probabilidad de infección. Una revisión sistemática encontró que la técnica del "ojal" se asocia con mayor riesgo de infecciones relacionadas con la fístula arteriovenosa, siendo la tasa de infección de 0.39 eventos por 1000 días de punción con la técnica del "ojal" frente a 0,10 eventos por 1000 días de punción con la técnica "en escalera". No obstante, esta tasa es inferior a las tasa de infecciones ocasionadas por los catéteres venosos centrales encontrada en un artículo de cohortes (1,27/1000 días de catéter) ${ }^{33}$.

\section{Resultados clínicos}

La mayoría de estudios encontrados comunican una mejora de los resultados clínicos del paciente en HDD, cuando se realiza a diario o al menos 5 veces por semana (Resumen en Tabla 3) $)^{10,17,19,20,34}$. generando a su vez preocupación al paciente ${ }^{19}$, apuntando algunos autores que si el paciente contara con cuidadores remunerados se reduciría la carga de estos familiares 10. Esta sobrecarga del cuidador, es destacada por algunos autores como responsable de la disminución de la adherencia a la técnica ${ }^{24}$. Algún artículo apunta como ayuda importante para
Tabla 3. Resultados clínicos asociadas a la HDD diaria o intensiva.

\section{Resultados clínicos de la HDD}

Mejor control de toxinas urémicas, con mejor tolerancia en las sesiones, eliminando el síndrome de fatiga posdiálisis.

Mejor control de la anemia con menor dosis de estimulantes eritropoyéticos.

Mejor control de alteraciones óseo-minerales asociadas a la ERC con menor dosis de quelantes. Mejor control de parámetros nutricionales.

Mejor control de la tensión arterial con menor dosis de fármacos hipotensores, facilitando el control del peso y mantenimiento de la función renal residual.

Mejoría en parámetros cardiológicos como reducción de la hipertrofia ventricular izquierda.

Menor morbi-mortalidad. 
No se encontraron artículos que reflejaran mejoras clínicas de la HDD convencional (3 sesiones/semana) respecto a la misma pauta de HD en centro.

\section{Supervivencia de HDD}

En el análisis de la supervivencia, no existen mucha bibliografía y los datos están referidos a pocos artículos. Un estudio comunica que la HDD se asocia con un riesgo $13 \%$ menor de mortalidad por todas las causas que la HD en centro sanitario. Estos datos sugieren que, en relación con la HD en el centro tres veces por semana, la HDD se asocia con modestas mejoras en la supervivencia ${ }^{35}$.

En comparación con los pacientes con trasplante renal funcionante, los pacientes canadienses que reciben HDD intensiva (16 horas por semana) tienen una supervivencia comparable a la de los receptores de trasplante de riñón de donante fallecido en los Estados Unidos. En esta cohorte, el trasplante de riñón se asoció con mayor supervivencia del paciente, pero mayores tasas de hospitalización temprana, en comparación con HHD intensiva ${ }^{36}$.

En otro artículo se destaca que la HDD diaria se asoció con menor riesgo de mortalidad que la DP domiciliaria, menor tasa de hospitalizaciones (HR 0,73; IC del $95 \%: 0,67-0,79 ; p<0,001)$ y menos días de hospitalización $(5,2 \text { frente a 9,2 días/paciente-año; } p<0,001)^{37}$. Otro artículo destaca que la HHD diaria se asoció con un riesgo $20 \%$ menor de mortalidad por todas las causas y un $37 \%$ menor riesgo de fracaso de la técnica, en comparación con la DP38. Además, también se ha señalado mejor supervivencia del paciente en HDD diaria a 5 años ( $85 \%$ versus $44 \%$ ) en comparación con la DP39.

\section{Discusión}

La HDD es una técnica de HD poco utilizada en la mayoría de los países excepto en Australia y Nueva Zelanda31, con una prevalencia muy baja entre las distintas modalidades de TRS. Incluso en los países, que históricamente han tenido mayor prevalencia de HDD, han sufrido un descenso de estas en los últimos años ${ }^{40}$.

No existe una única explicación a esta tendencia por lo que su análisis resulta complejo, y comienza en las particularidades sociales, culturales, económicas y del sistema sanitario de cada país, y tiene que ver con la concepción de las TRS en el domicilio. En efecto, cuando se analiza la poca implantación de la diálisis peritoneal ambulatoria en el mundo, salen a relucir variables que para nada tiene que ver con la indicación médica u opción terapéutica libremente elegida por los pacientes ${ }^{41}$.

Por otro lado, hay que tener en cuenta, que realizar un procedimiento técnicamente complejo, como es la HD en el domicilio del paciente, no es sencillo sin una formación adecuada del paciente y familiar colaborador ${ }^{42}$. A esto hay que añadir, que los monitores convencionales de $H D$, necesitan una serie de requisitos adicionales que hay que instalar en el domicilio del paciente, para poder llevar a cabo un tratamiento correcto (tratamiento de agua, espacio físico, etc). Por ello, simplificar el diseño de las máquinas de HD para tratamientos domiciliarios podría alentar a más pacientes a elegirla, pues hasta ahora la HDD se ha realizado con monitores de HD convencionales30. Sin embargo, esta dificultad técnica no explica la poca utilización de la técnica sobre todo en países con gran desarrollo de la HD como España. Recientemente ha aparecido en el mercado un monitor especialmente diseñado para HDD, de fácil manejo, que no requiere de instalación de agua tratada y que puede ser manejado directamente por el propio paciente sin ayuda de otra persona, imitando el modelo de cicladoras que se utilizan en la DP31.

Por otro lado, es evidente que si la administración sanitaria quiere fomentar esta técnica, tiene que desarrollar políticas activas que fomenten su utilización por los pacientes con ERCA que precisan una TRS, y que, los diferentes servicios de Nefrología ofrezcan esta modalidad dialítica, por sus buenos resultados clínicos, porque resulta a largo plazo más barata, y sobre todo, porque supone una elección del paciente que decide libremente y hay que disponer de ella; aportando además, mayor flexibilidad en su vida diaria, mejora de la autonomía, la posibilidad de viajar o de organizar horarios laborales ${ }^{43,44}$. Para ello, es fundamental que los propios profesionales sanitarios implicados en la comunicación/divulgación de la HDD crean en ella.

La supervivencia de los pacientes que se dializan en HDD intensiva o diaria, es otro de los factores dignos de resaltar ${ }^{45}$. Frente a otras modalidades como son la HD en el centro o la diálisis peritoneal, la HDD diaria o intensiva presenta mejores resultados, si bien las series publicadas son pequeñas respecto a las de las otras modalidades. En un artículo se ha destacado que esta mejor supervivencia puede ser debido a un mejor control del fósforo sérico asociado con mayores dosis de diálisis ${ }^{46}$. 
Como mayor inconveniente, se ha destacado el aumento de la probabilidad de que el paciente contraiga infecciones, especialmente relacionadas con el acceso vascular, bien relacionado con el uso de catéteres 0 relacionado con la técnica de punción en "ojal". Por lo que el papel de enfermería en la prevención de este problema es crucial. El uso de antimicrobianos tópicos, el reciclaje continuo, la revisión de la técnica y las visitas domiciliarias desempeñan un papel fundamental en la prevención de las infecciones relacionadas con el acceso vascular ${ }^{23}$.

Otro aspecto destacado como inconveniente, es la sobrecarga del cuidador principal o familiar responsable junto con el paciente del desarrollo de la técnica en el domicilio. En efecto, la situación que rodea a los pacientes en HDD, puede generar una sobrecarga en los cuidadores, puesto que muchos de ellos, manifiestan estrés, miedos e inseguridad25,29. Para mejorar esta dificultad, se han propuesto medidas como, el uso de una sala virtual que permita a los pacientes estar en contacto con los profesionales sanitarios, lo que podría suponer además, la disminución de los eventos adver$\operatorname{sos}^{47}$.

\section{Limitaciones del estudio}

La primera limitación ha sido la propia búsqueda en sí, ya que encontramos abundante bibliografía sobre las ventajas/desventajas de la técnica, pero muy poca sobre el resto de variables de interés para esta revisión. Otra limitación, es sin duda, que la mayoría de los estudios revisados, aunque establecen conclusiones, recomiendan realizar más investigaciones sobre cada una de las variables analizadas, es decir para poder establecer unos hallazgos más concluyentes, es necesario que la técnica se introduzca más en todos los países y se obtengan datos de series más grandes.

\section{Consideraciones prácticas}

Es fundamental la divulgación de esta modalidad de TRS, porque desde el conocimiento de la misma por parte de los pacientes con ERCA, es cuando su elección será una realidad. En todo ello, puede y debe tener un papel activo la enfermería de las unidades de diálisis, como educadores sanitarios del paciente, ayudándole a tomar decisiones compartidas, a la hora de elegir modalidad de procedimiento dialítico.

A la vista de los resultados de esta revisión, podemos afirmar que:

- La HDD es una modalidad de TRS que como principales ventajas se destaca la mayor libertad y flexibi- lidad para que el paciente dirija su propio tratamiento, lo que le va a permitir más posibilidades laborales, de viajar y de ocio. Como principal inconveniente, además de tratarse de un tratamiento complejo que requiere espacio e instalación en el domicilio del paciente, se ha encontrado una mayor probabilidad de padecer infecciones, relacionadas con el acceso vascular, cuando se utilizan CVC o la técnica del "ojal", como técnica de punción de la fístula arteriovenosa nativa.

- Los resultados clínicos de HDD son similares a los de la HD en centros sanitarios. Sin embargo, cuando se realiza HDD diaria o intensiva (mínimo 5 sesiones a la semana) los resultados clínicos se muestran superiores a los de la HD en centros sanitarios y a la DP, siendo comparables a los del TR.

- Tanto en la implantación, enseñanza y seguimiento de la HDD es fundamental el papel de enfermería, ya que en todos los programas se destaca la intervención directa de enfermería en la captación de los pacientes desde las consultas de ERCA, en el proceso clave de la enseñanza al paciente y familiar propiciando que posteriormente sean autónomos en su domicilio, y finalmente en el seguimiento de los mismos, tanto a nivel de consulta como en visitas domiciliarias que refuercen la autonomía del paciente y ayuden al éxito de la técnica.

\section{El autor declara que no hay conflicto de interés.}

$$
\begin{gathered}
\text { Recibido: } 28 \text { diciembre } 2017 \\
\text { Revisado: } 10 \text { enero } 2018 \\
\text { Modificado: } 15 \text { enero } 2018 \\
\text { Aceptado: } 30 \text { enero } 2018
\end{gathered}
$$

\section{Bibliografía}

1. Soriano Cabrera S. Definición y clasificación de los estadios de la enfermedad renal crónica. Prevalencia. Claves para el diagnóstico precoz. Factores de riesgo de la enfermedad renal crónica. Nefrología. 2004; 24 Supl 6:27-4.

2. Otero González A, De Francisco A, Gayoso $P$, García F. Prevalence of chronic renal disease in Spain: Results of the EPIRCE study. Nefrología. 2010; 30(1): 78-6. 
3. Acosta Hernández PA, Chaparro López LC, Rey Anaconda CA. Quality of life and coping strategies in patients with chronic renal failure undergoing hemodialysis, peritoneal dialysis or kidney transplantation. Revista Colombiana de Psicología. 2008; (17): 9-26.

4. Malheiro Olivieira P, Arruda Soares D. Percepciones de las personas con insufiencia renal crónica sobre la calidad de vida. Enfermería global. 2012; 12 (1): 26-0.

5. Rebollo Rubio A, Morales Asensio JM, Pons Raventos MA, Mansilla Francisco JJ. Revisión de estudios sobre calidad de vida relacionada con la salud en la enfermedad renal crónica avanzada en España. Revista Nefrología. 2015; 35:92-09.

6. Registro de Diálisis y Trasplante de 2015. Sociedad Española de Nefrología. [Consultado 27 marzo 2017]. Disponible en: http://www.senefro.org/ modules. php?name=webstructure\&idwebstructure $=29$.

7. ALM de Francisco. El futuro del tratamiento de la enfermedad renal crónica. Nefrología. 2010; 30 (1): 1-9.

8. Registry annual reports. ERA-EDTA of 2014. [Consultado 28 marzo2017]. Disponible en: http://www.era-edta-reg.org/index.jsp?p=14.

9. Walker RC, Howard K, Morton RL. Home hemodialysis: a comprehensive review of patient-centered and economic considerations. Clinicoe con Outcomes Res. 2017; 9:149-61. D0I: 10.2147/ CEOR.S69340.

10. Karkar A, Hegbrant J, Strippoli GFM. Benefits and implementation of home hemodialysis: A narrative review. Saudi J Kidney Dis Transpl. 2015 Nov; 26(6):1095-07.

11. Pérez-Alba A, Barril-Cuadrado G, Castellano-Cerviño I, Martín-Reyes G, Pérez-Melón C, Slon-Roblero $F$, et al. Hemodiálisis domiciliaria en España. Nefrología (Madrid). 2015; 35(1):1-5.

12. Andreu Périz D, Moreno Arroyo C, Hidalgo Blanco MA. Situación actual de la hemodiálisis domiciliaria. Enferm Nefrol. 2016; 19(2):165-69.
13. Barril G, Sánchez Tomero JA. La hemodiálisis domiciliaria alternativa posible y necesaria. Nefrología. 2007; 27 (1).

14. Renau EM, Cerrillo V, Folch $M^{\mathrm{a}} \mathrm{J}$, Bort J, Salvador $M^{\mathrm{a}} \mathrm{C}$, Ronda $\mathrm{AC}$. Hemodiálisis domiciliaria diaria en nuestro hospital. Una opción de futuro. Enferm Nefrol. 2014; 17 Suppl (1): 116-40.

15. Mendes KDS, Silveira RCCP, Galvão CM. Revisão integrativa: método de pesquisa para a incorporação de evidencia na saúde e na enfermagem. Texto \& contexto enferm. 2008; 17(4): 758-64.

16. Whittemore R, Knafl K, Gray EN. The integrative review: updated methodology. J Adv Nurs. 2005; 52(5): 546-53.

17. Tennankore K, Nadeau-Fredette AC, Chan CT. Intensified home hemodialysis: clinical benefits, risks and target populations. Nephrol Dial Transplant. 2014; 29(7):1342-9.

18. Walker RC, Howard $\mathrm{K}$, Morton RL, Palmer SC, Marshall MR, Tong A. Patient and caregiver values, beliefs and experiences when considering home dialysis as a treatment option: a semi-structured interview study. Nephrol Dial Transplant. 2016; 31(1):133-41.

19. Tennankore KK, Chan CT, Curran SP. Intensive home haemodialysis: benefits and barriers. Nat Rev Nephrol. 2012; 8(9):515-22.

20. Trinh E, Chan CT. The Rise, Fall, and Resurgence of Home Hemodialysis. Semin Dial. 2017; 30(2):174-80.

21. Walker RC, Hanson CS, Palmer SC, Howard K, Morton RL, Marshall MR, et al. Patient and Caregiver Perspectives on Home Hemodialysis: A Systematic Review. Am J Kidney Dis. 2015 Mar $1 ; 65(3): 451-63$.

22. Schachter ME, Chan CT. Current state of intensive hemodialysis: a comparative review of benefits and barriers. Nephrol Dial Transplant. 2012; 27(12):4307-13.

23. Poon CK, Chan CT. Home hemodialysis associated infection-The "Achilles' Heel" of intensive hemodialysis. Hemodial Int. 2017; 21(2):155-60. 
24. Tejada-Tayabas LM, Partida-Ponce KL, Hernández-Ibarra LE. Coordinated hospital-home care for kidney patients on hemodialysis from the perspective of nursing personnel. Rev Lat Am Enfermagem. 2015; 23(2):225-33.

25. Tong A, Palmer S, Manns B, Craig JC, Ruospo M, Gargano $L$, et al. The beliefs and expectations of patients and caregivers about home haemodialysis: an interview study. BMJ open. 2013; 3(1).

26. Hanson CS, Chapman JR, Craig JC, Harris DC, Kairaitis LK, Nicdao M, et al. Patient experiences of training and transition to home haemodialysis: a mixed-methods study. Nephrology. 2016.

27. Young BA, Chan C, Blagg C, Lockridge R, Golper $T$, Finkelstein $F$, et al. How to overcome barriers and establish a successful home HD program. Clin J Am Soc Nephrol. 2012; 7(12):2023-32.

28. Appleby S. Shared Care, Home Haemodialysis and the Expert Patient. J Ren Care, 2013; 39 (Sup 1):16-1.

29. Welch JL, Thomas-Hawkins $C$, Bakas $T$, McLennon SM, Byers DM, Monetti CJ, et al. Needs, Concerns, Strategies, and Advice of Daily Home Hemodialysis Caregivers. Clin Nurs Res. 2014; 23(6):644-63.

30. Rajkomar A, Farrington K, Mayer A, Walker D, Blandford A. Patients' and carers' experiences of interacting with home haemodialysis technology: implications for quality and safety. BMC Nephrol. 2014; 15:195.

31. Nearhos J, Van Eps C, Connor J. Psychological factors associated with successful outcomes in home haemodialysis. Nephrology. 2013; 18 (7):505-9.

32. System One Portable Hemodialysis Machine|NxStage. NxStage Medical Inc. 2017 [Consultado 30 marzo 2017]. Disponible en: http://www.nxstage. $\mathrm{com} /$ patients/system-one/.

33. Muir CA, Kotwal SS, Hawley CM, Polkinghorne K, Gallagher MP, Snelling P, et al. Buttonhole cannulation and clinical outcomes in a home hemodialysis cohort and systematic review. Clin J Am Soc Nephrol. 2014; 9(1):110-9.
34. Marshall MR, Chan CT. The Evolution of Home HD - Meeting Modern Patient Needs. Contrib Nephrol. 2017; 189:36-45.

35. Weinhandl ED, Liu J, Gilbertson DT, Arneson TJ, Collins AJ. Survival in Daily Home Hemodialysis and Matched Thrice-Weekly In-Center Hemodialysis Patients. JASN. 2012; 23(5):895-904.

36. Tennankore KK, Kim SJ, Baer HJ, Chan CT. Survival and hospitalization for intensive home hemodialysis compared with kidney transplantation. $J$ Am Soc Nephrol. 2014; 25(9):2113-20.

37. Nesrallah GE, Li L, Suri RS. Comparative effectiveness of home dialysis therapies: a matched cohort study. Can J Kidney Health Dis. 2016; 3: 19.

38. Weinhandl ED, Gilbertson DT, Collins AJ. Mortality, Hospitalization, and Technique Failure in Daily Home Hemodialysis and Matched Peritoneal Dialysis Patients: A Matched Cohort Study. Am J Kidney Dis. 2016; 67(1):98-110.

39. Nadeau-Fredette AC, Hawley CM, Pascoe EM, Chan CT, Clayton PA, Polkinghorne KR, et al. An Incident Cohort Study Comparing Survival on Home Hemodialysis and Peritoneal Dialysis (Australia and New Zealand Dialysis and Transplantation Registry). Clin J Am Soc Nephrol. 2015; 10(8):1397-407.

40. Walker RC, Morton RL, Tong A, Marshall MR, Palmer S, Howard K. Patient and caregiver preferences for home dialysis-the home first study: a protocol for qualitative interviews and discrete choice experiments. BMJ Open. 2015; 5(4):e007405.

41. Caro Domínguez C, Garrido Pérez L, Sanz Turrado $M$. Influencia de la consulta de enfermedad renal crónica avanzada en la elección de modalidad de terapia renal sustitutiva. Enferm Nefrol. 2016; 19.(4):318-29.

42. Verhallen A. Successful Vascular Access Management for Home Haemodialysis: A Practical Approach. J Ren Care. 2013; 39(Sup 1):28-4.

43. Lorenzen JM, Thum $T$, Eisenbach GM, Haller $\mathrm{H}$, Kielstein JT. Conversion from conventional in-centre thrice-weekly haemodialysis to short 
daily home haemodialysis ameliorates uremia-associated clinical parameters. Int Urol Nephrol. 2012; 44(3):883-90.

44. Tennankore KK, d'Gama C, Faratro R, Fung $S$, Wong $E$, Chan CT. Adverse Technical Events in Home Hemodialysis. Am J Kidney Dis. 2015; 65(1):116-21.

45. Vega A, Sequí MJ, Abad S, Yuste C, Santos A, Macías N, et al. Daily Home Hemodialysis Is an Available Option for Renal Replacement Therapy in Spain. Ther Apher Dial. 2016; 20(4):408-12.

46. Rydell H, Clyne N, Segelmark M. Home- or Institutional Hemodialysis? a Matched Pair-Cohort Study Comparing Survival and Some Modifiable Factors Related to Survival. Kidney Blood Press Res. 2016; 41(4):392-01.

47. Raphael MJ, Nadeau-Fredette AC, Tennankore $\mathrm{KK}$, Chan CT. A virtual ward for home hemodialysis patients - a pilot trial. Can J Kidney Health Dis. $2015 ; 2: 37$.

Este artículo se distribuye bajo una Licencia Creative Commons Atribución-NoComercial 4.0 Internacional. https://creativecommons.org/licenses/by-nc/4.0/ 